\title{
RINGS OF FUNCTIONS IN LIPSCHITZ TOPOLOGY
}

\author{
JOUNI LUUKKAINEN
}

\section{Introduction}

Let $X$ and $Y$ be metric spaces with metrics $d$ and $d^{\prime}$, respectively. A map $f: X \rightarrow Y$ is Lipschitz if there is $L \geqq 0$ such that $d^{\prime}(f(x), f(y)) \leqq L d(x, y)$ for all $x, y \in X$. The smallest such $L$ is the Lipschitz constant $\operatorname{lip} f$ of $f$. These notions make sense also for pseudometric spaces. If each point of $X$ has a neighborhood on which $f$ is Lipschitz, $f$ is locally Lipschitz (LIP). If $f$ is bijective and both $f$ and $f^{-1}$ are Lipschitz (resp. LIP), $f$ is a Lipschitz homeomorphism (resp. lipeomorphism). See [14] for a more comprehensive treatment of Lipschitz topology.

This paper is concerned with real-valued Lipschitz and LIP functions on metric spaces. Theorem 1.2 characterizes the pairs $(\operatorname{Lip}(X), \operatorname{lip})$ and $(L(X), \operatorname{lip} \mid L(X))$, where $\operatorname{Lip}(X)$ and $L(X)$ are the sets of all Lipschitz functions and all bounded Lipschitz functions, respectively, on $X$ and where lip is the function $f \mapsto \operatorname{lip} f$ on Lip $(X)$. In Section 2 we investigate how homomorphisms of rings $L(X)$ are related to maps of metric spaces. In particular, we show in 2.23 that $L(X)$ and $L(Y)$ are isomorphic if and only if the completions of $X$ and $Y$ are LS homeomorphic in the sense of 2.14; this generalizes a result due to Sherbert [20]. Section 3 is devoted to the rings $l(X)$ and $l^{*}(X)$ of all LIP functions and all bounded LIP functions, respectively, on $X$. In 3.5 a new proof is given for Su's theorem [24,6.4'] that $X$ and $Y$ are lipeomorphic if and only if $l(X)$ and $l(Y)$ are isomorphic. A similar result holds for $l^{*}(X)$. In 3.11 we study relations between $X$ and a natural topology on $l(X)$. We also consider ideals of $l(X)$ and $l^{*}(X)$. In the last section we study ideals in and characterize homomorphisms of the rings $l_{0}(X)$ and $l_{00}(X)$ of all LIP functions on a locally compact metric space $X$ vanishing at infinity or with compact support, respectively, and obtain analogues of Su's theorem.

We will often use McShane's theorem [15, Theorem 1] stating that if $A \subset X$, then every Lipschitz $f: A \rightarrow R$ has an extension $\bar{f}: X \rightarrow R$ with $\operatorname{lip} \bar{f}=\operatorname{lip} f$. If $f, g: X \rightarrow R$ are Lipschitz and $h$ is either $\max (f, g)$ or $\min (f, g)$, then $\operatorname{lip} h \leqq$ $\max (\operatorname{lip} f, \operatorname{lip} g)$. Thus, if $f$ in McShane's theorem is bounded, $\bar{f}$ can be chosen to be bounded with the sup norm $\|\bar{f}\|_{\infty}=\|f\|_{\infty}$. 


\section{A characterization of the set of Lipschitz functions}

1.1. The characterization of ( $\operatorname{Lip}(X)$, lip) in 1.2 is based on the identity $f(x)=$ $\sup _{y \in X}(f(y)-(\operatorname{lip} f) d(x, y))$, valid for all $f \in \operatorname{Lip}(X)$ and $x \in X$ (cf. below). We denote $m(S)=\left\{f \in R^{S} \mid f\right.$ bounded $\}$ if $S$ is a set and sometimes $\operatorname{lip}_{d} f=\operatorname{lip} f$ if $f \in \operatorname{Lip}(X)$. If $S$ is a set, $K \subset R^{S}$, and $\max (f, g) \in K$ for all $f, g \in K$, we say that $K$ is max-closed.

1.2. Theorem. Let $S$ be a nonvoid set, let $A \subset R^{S}$ (resp. $A \subset m(S)$ ) and let $p: A \rightarrow[0, \infty)$. Then there is a metric $\varrho$ on $S$ such that $A=\operatorname{Lip}(S, \varrho)(\operatorname{resp} . A=L(S, \varrho))$ and $p(f)=\operatorname{lip}_{e} f$ for every $f \in A$ if and only if the following six conditions are satisfied, where $A_{t}=\{f \in A \mid p(f) \leqq t\}$ and $A_{t}(x)=\left\{f \in A_{t} \mid f(x)=0\right\}$ for $t \geqq 0$ and $x \in S$ :

(1) If $f \in A$ and $t \in R$, then $t f \in A$ and $p(t f)=|t| p(f)$.

(2) If $f \in A$ and $t \in R$, then $f+t \in A$ and $p(f+t)=p(f)$.

(3) If $f, g \in A$ and $h=\max (f, g)$, then $h \in A$ and $p(h) \leqq \max (p(f), p(g))$.

(4) If $K \subset A$ is max-closed and nonvoid, $M=\sup \{p(g) \mid g \in K\}<\infty$, and $f=\sup K<\infty \quad$ (resp. $f$ is bounded), then $f \in A$ and $p(f) \leqq M$.

(5) There is $x_{0} \in S$ with $\sup \left\{f(x) \mid f \in A_{1}\left(x_{0}\right)\right\}<\infty$ for every $x \in S$.

(6) A separates points of $S$ or, if card $S=1$, is nonvoid.

The metric @ is uniquely given by

$$
\varrho(x, y)=\sup \left\{|f(x)-f(y)| \mid f \in A_{1}\right\}, \quad x, y \in S .
$$

Proof. We consider the case of bounded functions only, because the case of not necessarily bounded functions is similar and simpler. Let $\varrho$ be a metric on $S$ and let $A=L(S, \varrho)$ and $p=\operatorname{lip}_{\varrho} \mid A$. It is easy to see that the conditions (1), .., (5) hold and that $x_{0}$ can be chosen arbitrarily. If $x, y \in S$ and $f(z)=\min (\varrho(x, z), \varrho(x, y))$, $z \in S$, then $f \in A_{1}$ and $|f(x)-f(y)|=\varrho(x, y)$. Thus (6) is satisfied and, if $e(x, y)$ is the right-hand side of (1.3), $e \geqq \varrho$. Since obviously $e \leqq \varrho,(1.3)$ follows.

Now we consider $A \subset m(S)$ and $p: A \rightarrow[0, \infty)$ satisfying (1), ., (6). Since $A_{1} \neq \emptyset$ by (6) and (1), one can define a function $\varrho: S \times S \rightarrow[0, \infty]$ by (1.3). Let $x, y \in S$. If $f \in A_{1}$, then $g=f-f\left(x_{0}\right) \in A_{1}\left(x_{0}\right)$ by (2) and $|g(x)-g(y)|=|f(x)-f(y)|$. Thus $\varrho(x, y)=\sup \left\{|g(x)-g(y)| \mid g \in A_{1}\left(x_{0}\right)\right\}$. Since $-A_{1}\left(x_{0}\right) \subset A_{1}\left(x_{0}\right)$ by (1), this and (5) imply $\varrho(x, y)<\infty$. Therefore $\varrho$ is a pseudometric on $S$. If $x \neq y$, there is, by (6) and (1), $f \in A_{1}$ with $f(x) \neq f(y)$, whence $\varrho(x, y) \geqq|f(x)-f(y)|>0$. Thus $\varrho$ is a metric.

Let $f \in A$. We show that $\operatorname{lip}_{e} f \leqq p(f)$. Let first $p(f)=0$. If $t>0$, then $p(t f)=$ $t p(f)=0$, whence $t f \in A_{1}$, and thus $t|f(x)-f(y)| \leqq \varrho(x, y)$ for all $x, y \in S$. This implies that $f$ is constant and $\operatorname{lip}_{\varrho} f=0=p(f)$. If $p(f)=1$, then $|f(x)-f(y)| \leqq$ $\varrho(x, y)$ for all $x, y \in S$, which implies $\operatorname{lip}_{\varrho} f \leqq 1=p(f)$. The general case now follows from (1).

The following facts are needed. If $f \in m(S)$ is constant, then, $g$ being any element of $A, f=0 g+f \in A$ and $p(f)=0 p(g)=0$ by (1) and (2). If $t \geqq 0$ and $f, g \in A_{t}$, then $\min (f, g) \in A_{t}$ by (1) and (3). 
Next we show that if $a \in S, t>0$, and $h(x)=\varrho(x, a)$ for each $x \in S$, then $f=\min (h, t) \in A_{1}$. First, we have as above $h=\sup A_{1}(a)$. Thus, if

$$
K=\left\{\min (g, t) \mid g \in A_{1}(a)\right\},
$$

then $K \subset A_{1}, f=\sup K$, and $K$ is max-closed since $A_{1}(a)$ is so. Hence $f \in A_{1}$ by (4).

Finally, let $f \in L(S, \varrho)$ and $M=\operatorname{lip}_{\varrho} f$. Then $K=\left\{g \in A_{M} \mid g \leqq f\right\}$ is max-closed. Using an idea due to the proof of [15, Theorem 1], we define $g_{y} \in m(S)$ for each $y \in S \quad$ by $\quad g_{y}(x)=f(y)-\min \left(M \varrho(x, y), 2\|f\|_{\infty}\right) ; \quad$ then $g_{y}(x) \leqq f(x)=g_{x}(x)$ and $g_{y} \in A_{M}$. Thus $f=\sup K$, and hence $f \in A_{M}$ by (4). This proves that $A=L(S, \varrho)$ and $p=\operatorname{lip}_{\varrho} \mid A$.

1.4. Remarks. 1) The uniqueness of the metric $\varrho$ in 1.2 is a well-known fact $[19$, p. 81].

2) We obtain a similar result for pseudometric spaces $(S, \varrho)$ by replacing $(6)$ in 1.2 by the condition $A \neq \emptyset$.

3) It is easy to see that the key condition (4) in 1.2 could be replaced by the condition that $A_{1}$ is closed in $R^{S}$ (resp. $m(S)$ ) with respect to the topology of pointwise convergence. It is not hard to show that (4) could also be replaced by the following condition: There is $x_{1} \in S$ such that if $K \subset A_{1}\left(x_{1}\right)$ is max-closed and nonvoid and $f=\sup K$ is finite (resp. bounded), then $f \in A_{1}$. In (2) we could fix $t=1$, and in (1) and (2) we could replace the equality by the inequality $\leqq$.

1.5. Examples. In 1.2 all the six conditions of each of the two characterizations are essential. We show this by constructing for every $i \in\{1, \ldots, 6\}$ a nonvoid set $S$, a set $A \subset m(S)$ and a function $p: A \rightarrow[0, \infty)$ with the following two properties.

(a) If $1 \leqq j \leqq 6$ and $j \neq i$, then $(j)$ holds. Here we do not assume $f$ to be bounded in (5).

(b) Let $\varrho$ be a metric on $S$. Then, if $i \neq 6$, it is not true that $p=\operatorname{lip}_{\varrho} \mid A$, or, if $i=6, A \neq L(S, \varrho)$ (and thus $A \neq \operatorname{Lip}(S, \varrho)$ ).

Let $I=[0,1] \subset R$.

$i=1: S=I, A=\operatorname{Lip}(I), p(f)=(\operatorname{lip} f)^{2}$.

$i=2: S=\{0,1\}, A=R^{S}, p(f)=\left|f(0)^{3}-f(1)^{3}\right|^{1 / 3}$.

$i=3: S=I, A=\operatorname{Lip}(I), p(f)=|f(0)-f(1)|+\operatorname{lip} f$. If $f, g \in A$ are defined by $f(x)=1-|2 x-1|$ and $g(x)=x$, then $p(f)=p(g)=2<3=p(\max (f, g))$; thus (b) holds.

$i=4$ : Let $S=I$. Let $A$ consist of all functions $f: I \rightarrow R$ such that there are a division $0=t_{0}<t_{1}<\ldots<t_{n}=1$ of $I$ and numbers $a_{k}, b_{k} \in R, 1 \leqq k \leqq n$, for which $f \mid\left[t_{k-1}, t_{k}\right]$ is given by $x \mapsto a_{k} x+b_{k}$ or $x \mapsto a_{k} x^{2}+b_{k}, 1 \leqq k \leqq n$. It is easy to see that $p(f)=\max \left\{\left|a_{k}\right| \mid 1 \leqq k \leqq n\right\}$ is well-defined. We prove (b). Define $f \in A$ by $f(x)=2 x^{2}$ if $x \leqq 4^{-1}$ and by $f(x)=8^{-1}$ otherwise; then $p(f)=2$. Let $K=\{g \in A \mid g$ piecewise- 
linear, $g \leqq f, p(g) \leqq 1\}$; then $K$ is max-closed. Since the derivative $f^{\prime}(x) \leqq 1$ if $x \leqq 4^{-1}$, it is easy to see that $f=\sup K$. Thus, if (b) does not hold, $p(f) \leqq 1$, which is a contradiction.

$$
\begin{aligned}
& i=5: S=\{0,1\}, A=R^{S}, p=0 . \\
& i=6: S=\{0,1\}, A=\{f \mid f: S \rightarrow R \text { constant }\}, \quad p=0 \text { (or } S=\{0\}, A=\emptyset, p=\emptyset \text { ). }
\end{aligned}
$$

1.6. Example. Let $S$ be a nonvoid set and $\omega: S \times S \rightarrow[0, \infty)$. We define $A=\operatorname{Lip}(S, \omega)$ and $p=\operatorname{lip}_{\omega}$ as if $\omega$ were a metric. Then it is easy to see that $(A, p)$ has the properties (1), .., (5) of 1.2 (the case of not necessarily bounded functions) and that $A \neq \emptyset$. Thus by 1.4.2 there is a pseudometric $\varrho$ on $S$ such that $\operatorname{Lip}(S, \omega)=$ $\operatorname{Lip}(S, \varrho)$ and $\operatorname{lip}_{\omega}=\operatorname{lip}_{\varrho}$. Johnson $[9,1.2]$ proved directly the corresponding result for bounded real- or complex-valued functions. The pseudometric $\varrho$ is the greatest pseudometric on $S$ with $\varrho \leqq \omega$.

1.7. Four functors. We define four maps $P_{*}, P^{*}, L_{*}$, and $L^{*}$ from the category $E_{0}$ of nonvoid sets and surjections into the category $E$ of sets and maps as follows. If $S$ is a nonvoid set, we let $P_{*}(S)=P^{*}(S)$ be the set of all pseudometrics on $S$ and $L_{*}(S)=L^{*}(S)$ the set of all pairs $(A, p)$ with $\emptyset \neq A \subset R^{S}$ and $p: A \rightarrow[0, \infty)$ satisfying the conditions (1), ., (5) of 1.2 (in the case of not necessarily bounded functions). By 1.4.2 there is a canonical bijection $J_{S}: P_{*}(S) \rightarrow L_{*}(S)$.

Let $S$ and $T$ be nonvoid sets and let $\varphi: S \rightarrow T$ be a surjection. We define maps $P_{*}(\varphi): P_{*}(S) \rightarrow P_{*}(T), \quad P^{*}(\varphi): P^{*}(T) \rightarrow P^{*}(S), \quad L_{*}(\varphi): L_{*}(S) \rightarrow L_{*}(T), \quad$ and $L^{*}(\varphi): L^{*}(T) \rightarrow L^{*}(S)$ as follows. If $\varrho \in P_{*}(S)$, we let $P_{*}(\varphi)(\varrho)(x, y)$ for $x, y \in T$ be the infimum of the sums $\sum_{i=1}^{n} \varrho\left(\varphi^{-1}\left(t_{i-1}\right), \varphi^{-1}\left(t_{i}\right)\right)$ where $n \geqq 1, t_{0}, \ldots, t_{n} \in T$, $t_{0}=x$, and $t_{n}=y$. If $\varrho \in P^{*}(T)$, we set $P^{*}(\varphi)(\varrho)(x, y)=\varrho(\varphi(x), \varphi(y))$ for $x, y \in S$. If $(A, p) \in L_{*}(S)$, we define $L_{*}(\varphi)(A, p)=\left(A_{*}, p_{*}\right)$ by setting $A_{*}=\left\{f \in R^{T} \mid f \varphi \in A\right\}$ and $p_{*}(f)=p(f \varphi)$ for $f \in A_{*}$. If $(A, p) \in L^{*}(T)$, we define $L^{*}(\varphi)(A, p)=\left(A^{*}, p^{*}\right)$ by setting $A^{*}=\{f \varphi \mid f \in A\}$ and $p^{*}(f \varphi)=p(f)$ for $f \in A$; since $\varphi$ is surjective, $p^{*}$ is well-defined. Note that $P_{*}(\varphi)$ and $P^{*}(\varphi)$ do not generally map metrics into metrics.

1.8. Theorem. The maps $P_{*}$ and $L_{*}$ are covariant functors and the maps $P^{*}$ and $L^{*}$ are contravariant functors $E_{0} \rightarrow E$. The family of the bijections $J_{S}$ defines natural equivalences $P_{*} \rightarrow L_{*}$ and $P^{*} \rightarrow L^{*}$. If $\varphi$ is a surjection, then $P_{*}(\varphi) P^{*}(\varphi)=\mathrm{id}$ and $L_{*}(\varphi) L^{*}(\varphi)=\mathrm{id}$.

Proof. The proof is a straightforward verification. If 1.4 .2 is assumed, the natural equivalence of $P_{*}$ and $L_{*}$ also follows from [12, 1.9-1.11].

1.9. Finally we give a version of 1.2 for $S$ a topological space. We say that $A \subset R^{S}$ separates points from closed sets if, whenever $F \subset S$ is closed and $x \in S \backslash F$, there is $f \in A$ with $f(x) \notin \overline{f(F)}$. Let $C(S)$ be the set of all real-valued continuous functions on $S$. It is easy to see that a metric $\varrho$ on $S$ is compatible with the topology of $S$ if and only if $\operatorname{Lip}(S, \varrho) \subset C(S)$ and $\operatorname{Lip}(S, \varrho)$ separates points from closed sets. 
1.10. Theorem. Let $S$ be a nonvoid topological space, let $A \subset R^{S}$ and let $p: A \rightarrow[0, \infty)$. Then there is a compatible metric @ on $S$ such that $A=\operatorname{Lip}(S, \varrho)$ and $p=\operatorname{lip}_{\varrho}$ if and only if the conditions (1), (2), (3), and (6) in 1.2 as well as the following four are satisfied:

(4a) Let $K \subset A_{1}$ be max-closed and nonvoid, let $f=\sup K$ be finite and continuous, and suppose that if $F \subset S$ is compact and $\varepsilon>0$, there is $g \in K$ with $g(x)>$ $f(x)-\varepsilon$ for each $x \in F$. Then $f \in A_{1}$.

(5a) There are $x_{0} \in S$ and a dense set $D \subset S$ with sup $\left\{f(x) \mid f \in A_{1}\left(x_{0}\right)\right\}<\infty$ for every $x \in D$.

(7) There is $x_{2} \in S$ with $A_{1}\left(x_{2}\right)$ equicontinuous.

(8) A separates points from closed sets.

Proof. We prove only the sufficiency. Since by (7) and (2) $A_{1}$ is equicontinuous, (5a) implies (5). Let $K \subset A_{1}$ be max-closed and nonvoid and let $f=\sup K<\infty$. Then $f \in \bar{K}$ in the topology of pointwise convergence. Since $K$ is equicontinuous, this implies that $f$ is continuous. If $F \subset S$ is compact and $\varepsilon>0$, it is easy to find $g \in K$ with $g(x)>f(x)-\varepsilon$ for each $x \in F$. Thus $f \in A_{1}$ by (4a), whence (4) holds. Now 1.2 gives a metric $\varrho$ on $S$ such that $A=\operatorname{Lip}(S, \varrho)$ and $p=\operatorname{lip}_{\varrho}$. Since $A \subset C(S)$, by (8) $\varrho$ is compatible.

1.11. Remarks. 1) Theorem 1.10 has obvious modifications for the cases of bounded functions, of compatible pseudometrics, and of continuous pseudometrics.

2) We can replace (4a) in 1.10 by the condition that $A_{1}$ is closed in $C(S)$ with respect to the topology of compact convergence. If $S$ is connected, then (7) implies (5), and hence (5a) can be omitted. If $S$ is compact, (8) can be omitted and (4a) can be replaced by the following condition. If a sequence $g_{n} \in A_{1}, g_{1} \leqq g_{2} \leqq \ldots$, converges uniformly to $f \in C(S)$, then $f \in A_{1}$.

\section{Rings of Lipschitz functions}

2.1. In this section we study the rings $L(X)$, but as an application we also obtain the result that $X$ and $Y$ are Lipschitz homeomorphic if and only if there is a homeomorphism of $\operatorname{Lip}(X)$ onto $\operatorname{Lip}(Y)$ in the topologies of pointwise convergence (or compact convergence) that defines an isomorphism of $L(X)$ onto $L(Y)$ (this follows from 2.27). The set $\operatorname{Lip}(X)$ itself is a ring only if $X$ is bounded or equivalently $\operatorname{Lip}(X)=L(X)$. By homomorphism we always mean ring homomorphism (homomorphisms of rings with unity are not assumed to carry unity to unity).

2.2. Lemma. Let $A$ be an algebra over $R$ (with or without unity and possibly nonassociative) and let $\varphi: A \rightarrow R$ be a ring homomorphism. Then $\varphi$ is linear.

Proof. Let $x \in A$ and $t \in R$. If $\varphi(x) \neq 0$, define $\alpha: R \rightarrow R$ by $\alpha(s)=\varphi(s x) / \varphi(x)$. Then $\alpha$ is a nonzero ring homomorphism, whence the identity [6, 0.22]. Thus $\varphi(t x)=$ 
$t \varphi(x)$. If $\varphi(x)=0$, then $\varphi(t x)=0=t \varphi(x)$, because otherwise $t \neq 0$ and hence by the above $\varphi(x)=t^{-1} \varphi(t x) \neq 0$.

2.3. Function rings. This paragraph is preliminary. The carrier space $A^{\circ}$ of a ring $A$ is the set of all nonzero homomorphisms $\varphi: A \rightarrow R$ with the weakest topology in which every function $\hat{x}: \varphi \mapsto \varphi(x), x \in A$, on this set is continuous.

Let $S$ be a set and let $A$ be a subring of $R^{S}$ which contains constants and is inverse-closed, i.e., if $f \in A$ with $|f| \geqq \varepsilon$ for some $\varepsilon>0$, then $1 / f \in A$. If $f \in A$ and $\varphi \in A^{\circ}$, then, because $\varphi$ is linear by 2.2 , it is easy to see that $\varphi(f) \in \overline{f(S)}$; hence $|\varphi(f)| \leqq\|f\|_{\infty}$ provided that $f$ is bounded.

Let $A$ be a subring of $C(X)$ that is completely regular, i.e., if $F \subset X$ is closed and $x \in X \backslash F$, there is $f \in A$ with $f \mid F=0$ and $f(x) \neq 0$. It is easy to see that if one defines $\varphi_{x} \in A^{\circ}$ for $x \in X$ by $\varphi_{x}(f)=f(x)$, then $x \mapsto \varphi_{x}$ is a topological embedding of $X$ into $A^{\circ}$. Therefore we may consider $X$ as a subspace of $A^{\circ}$ and $\hat{f}$ as a continuous extension of $f \in A$ to $A^{\circ}$. Let $C^{*}(X)=C(X) \cap m(X)$. Suppose now that $A \subset C^{*}(X)$ and that $A$ contains constants and is inverse-closed. Then $\|\varphi\|=1$ for each $\varphi \in A^{\circ}$ if we consider $A$ with the sup norm. Thus it can be proved as in [13, Theorem 19B and Corollary 19D] that $A^{\circ}$ is a Hausdorff compactification of $X$.

Let $A$ and $B$ be rings with unities 1 and $1^{\prime}$. Then every homomorphism $T: A \rightarrow B$ with $T(1)=1^{\prime}$ induces a continuous map $T^{\circ}: B^{\circ} \rightarrow A^{\circ}$ by $T^{\circ}(\varphi)=\varphi T$. Let $P$ and $Q$ be sets. Then every map $t: P \rightarrow Q$ induces a homomorphism $t^{\prime}: R^{Q} \rightarrow R^{P}$ by $t^{\prime}(f)=f t$, and $t^{\prime}(1)=1$; we denote by $t^{\prime}$ also all homomorphisms that $t^{\prime}$ defines between subrings.

2.4. The ring $L(X)$ is in fact an algebra, with the constant function 1 as unity, and by 2.2 the homomorphisms $L(X) \rightarrow L(Y)$ are algebra homomorphisms. Sherbert [20] studied the algebra $L(X, C)$ over $C$ of all bounded complex-valued Lipschitz functions on $X$. Since $L(X, C)$ is inverse-closed, $\varphi(L(X)) \subset R$ if $\varphi: L(X, C) \rightarrow C$ is an algebra homomorphism. This implies that every algebra homomorphism $T: L(X, C) \rightarrow L(Y, C)$ defines a homomorphism $T_{R}: L(X) \rightarrow L(Y)$. The map $T \mapsto T_{R}$ is a bijection of the set of all algebra homomorphisms $L(X, C) \rightarrow L(Y, C)$ onto the set of all homomorphisms $L(X) \rightarrow L(Y)$. Thus in studying homomorphisms the real and complex cases are not essentially different.

2.5. The carrier space $s X=L(X)^{\circ}$ of $L(X)$ is a Hausdorff compactification of $X$, because $L(X)$ is a completely regular, inverse-closed subalgebra of $C^{*}(X)$. With the norm

$$
\|f\|=\|f\|_{\infty}+\operatorname{lip} f
$$

$L(X)$ is a Banach algebra, and $\|1\|=1$ if $X \neq \emptyset$. By $2.3 s X$ lies on the unit sphere of the dual $L(X)^{*}$ of $L(X)$. This gives a metric $\sigma \leqq 2$ on $s X$. The carrier space topology of $s X$ is weaker than the metric topology. 
2.7. Sherbert considered the (complex) carrier space $\Sigma$ of the Banach algebra $L(X, C) ; 2.5$ is a real analogue of the corresponding points of [20]. By $2.4 \varphi_{R}=$ $\varphi \mid L(X)$ belongs to $s X$ for each $\varphi \in \Sigma$ and $\varphi \mapsto \varphi_{R}$ is a bijection $\Sigma \rightarrow s X$. This bijection is an equivalence of the compactifications $\Sigma$ and $s X$ of $X$ and it is an isometry. The nontrivial inequality in $\|\varphi-\psi\|=\left\|\varphi_{R}-\psi_{R}\right\|, \varphi, \psi \in \Sigma$, follows from the fact that if $f \in L(X, C)$ and $(\varphi-\psi)(f) \in R$, then $(\varphi-\psi)(f)=(\varphi-\psi)(\operatorname{Re} f)$ and $\|\operatorname{Re} f\| \leqq\|f\|$.

Next we investigate properties of $s X$.

2.8. Let $\tilde{X}$ be the completion of $X$. The inclusion map $j: X \rightarrow \tilde{X}$ induces an isometric isomorphism $j^{\prime}: L(\tilde{X}) \rightarrow L(X)$ (cf. [20, p. 1387]), and then $j^{\prime \circ}: s X \rightarrow s \tilde{X}$ is a homeomorphism (in the carrier space topologies) as well as an isometry and keeps the points of $X$ fixed. Thus we may identify $s X$ and $s \tilde{X}$ both as compactifications of $X$ and as metric spaces. Then $X \subset \tilde{X} \subset s X$.

In the following theorem we generalize this result.

2.9. Theorem. Let $A \subset X$. Then the compactifications $s A$ and $\bar{A} \subset s X$ of $A$ are equivalent. The equivalence $\alpha: s A \rightarrow \bar{A}$ is given by $\alpha(\varphi)(f)=\varphi(f \mid A), \varphi \in s A, f \in L(X)$, and $\alpha$ is an isometry.

Proof. The formula of $\alpha$ defines obviously a continuous map $\alpha: s A \rightarrow s X$ with lip $\alpha \leqq 1$. In fact, if $j: A \rightarrow X$ is the inclusion map, the homomorphism $j^{\prime}: L(X) \rightarrow$ $L(A)$ induces $\alpha$. If $f \in L(A)$, there is $g \in L(X)$ with $g \mid A=f$ and $\|g\|=\|f\|$. This implies that $\alpha$ is an isometric embedding. Clearly $\alpha \mid A=\mathrm{id}$. We conclude that $\alpha$ is a homeomorphism of $s A$ onto $\bar{A}$.

2.10. Lemma. If $A$ and $B$ are disjoint closed subsets of $s X$, there is $f \in L(X)$ such that $\hat{f}|A=0, \hat{f}| B=1$, and $0 \leqq \hat{f} \leqq 1$.

Proof. Choose $g \in C(s X)$ with $g \mid A=-1$ and $g \mid B=2$. Since $\{\hat{f} \mid f \in L(X)\}$ is a point-separating subring of $C(s X)$ containing constants, by the Stone-Weierstrass theorem there is $h \in L(X)$ such that $\|\hat{h}-g\|_{\infty} \leqq 1$. Define $f=\min (\max (h, 0), 1)$.

2.11. Corollary. Two subsets $A$ and $B$ of $X$ have disjoint closures in $s X$ if and only if $d(A, B)>0$.

Proof. The following conditions are consecutively equivalent: $d(A, B)>0$; there is $f \in L(X)$ with $f \mid A=0$ and $f \mid B=1$; there is $f \in L(X)$ with $\hat{f} \mid \bar{A}=0$ and $\hat{f} \mid \bar{B}=1$; and, by $2.10, \bar{A} \cap \bar{B}=\emptyset$. The closures are taken in $s X$.

2.12. Lemma. A point of $s X$ belongs to $\tilde{X}$ if and only if it has a countable neighborhood base.

Proof. We may assume that $\tilde{X}=X$. If $x \in s X \backslash X$ has a countable neighborhood base, there is a sequence $\left(x_{n}\right)$ in $X$ converging to $x$. Since $X$ is complete and no subsequence of $\left(x_{n}\right)$ converges in $X$, the subset $\left\{x_{1}, x_{2}, \ldots\right\}$ of $X$ is not totally bounded, 
and thus there is a subsequence $\left(y_{j}\right)$ of $\left(x_{n}\right)$ with $\inf _{i \neq j} d\left(y_{i}, y_{j}\right)>0$. Now the fact $\lim y_{2 j-1}=x=\lim y_{2 j}$ contradicts 2.11 . The proof is completed by the fact that if a dense subspace of a regular space is first countable at a point $x$, then the whole space is first countable at $x$; cf. $[6,9.7]$.

2.13. Remark. Lemma 2.10 is an improvement of [20, Corollary 4.3]. Corollary 2.11 shows that $s X$ is the Samuel--Smirnov compactification of $X$ defined in [22] for every separated proximity space. The equivalence of the two compactifications in 2.9 holds more generally for separated proximity spaces [22, Proof of Theorem 15]. Lemma 2.12 is the same as [23, Theorem 7], a corollary of a more general result.

2.14. Definition. A map $f: X \rightarrow Y$ is Lipschitz in the small (abbreviated LS) if there are $\varepsilon>0$ and $L \geqq 0$ such that $d^{\prime}(f(x), f(y)) \leqq L d(x, y)$ for all $x, y \in X$ with $d(x, y)<\varepsilon$. If $f$ is a bijection and both $f$ and $f^{-1}$ are LS, then $f$ is an LS homeomorphism. Two metrics $d_{1}, d_{2}$ on a set $S$ are LS equivalent if id: $\left(S, d_{1}\right) \rightarrow\left(S, d_{2}\right)$ is an LS homeomorphism.

2.15. Every Lipschitz map is LS. Every bounded LS map is Lipschitz. Every LS map is LIP and uniformly continuous. The composition of two LS maps is LS. The bounded metrics $d /(1+d)$ and $\min (d, 1)$ are LS equivalent to $d$. It follows that $f: Y \rightarrow X$ is LS if and only if $f: Y \rightarrow(X, d /(1+d))$ is Lipschitz.

2.16. Lemma. The metrics $d$ and $\sigma \mid X$ on $X$ are LS equivalent. In fact, $d /(1+d) \leqq \sigma \mid X \leqq d$.

Proof. The definition of $\sigma$ at once implies $\sigma \mid X \leqq d$. Let $x, y \in X$ and define $f \in L(X)$ by $f(z)=\min (d(z, y), d(x, y)) /(1+d(x, y))$. Then, since $\|f\| \leqq 1, \sigma(x, y) \geqq$ $|f(x)-f(y)|=d(x, y) /(1+d(x, y))$.

In the next theorem we characterize the homomorphisms $T: L(X) \rightarrow L(Y)$ for arbitrary metric spaces $X$ and $Y$.

2.17. Theorem. Let $T: L(X) \rightarrow L(Y)$ be a homomorphism. Then there are a unique set $E \subset Y$ with $d^{\prime}(E, Y \backslash E)>0$ (if $E \neq \emptyset \neq Y \backslash E$ ) and a unique Lipschitz map $t: E \rightarrow s X$ such that

$$
T(f)(x)=\left\{\begin{array}{lll}
\hat{f}(t(x)) & \text { if } & x \in E \\
0 & \text { if } & x \in Y \backslash E
\end{array} \quad(f \in L(X)) .\right.
$$

Conversely, if $E$ and $t$ are as above, then (2.18) defines a homomorphism $T: L(X) \rightarrow$ $L(Y)$.

Proof. Let $T: L(X) \rightarrow L(Y)$ be a homomorphism. Let $E=T(1)^{-1}(1)$. Since $T(1) \mid Y \backslash E=0$, we have $d^{\prime}(E, Y \backslash E)>0$ and $T(f) \mid Y \backslash E=0$ for each $f \in L(X)$. Let $T_{1}: L(X) \rightarrow L(E)$ be the homomorphism $f \mapsto T(f) \mid E$; then $T_{1}$ is linear and $T_{1}(1)=1$. Hence $T_{1}$ induces a map $T_{1}^{\circ}: s E \rightarrow s X$. We consider $L(X)$ and $L(E)$ as Banach algebras with the norm (2.6). Then one can prove as in the proof of [13, 
Theorem 24B] that $T_{1}$ is continuous. Therefore $T_{1}$ has a bounded linear dual map $T_{1}^{*}: L(E)^{*} \rightarrow L(X)^{*}$. Thus $T_{1}^{\circ}=T_{1}^{*} \mid s E$ is Lipschitz. Since the inclusion map $j: E \rightarrow s E$ is also Lipschitz (2.16), $t=T_{1}^{\circ} j: E \rightarrow s X$ is Lipschitz. The formula (2.18) is easy to verify. The uniqueness result is trivial.

Conversely, let $E$ and $t$ be given. If $f \in L(X)$, we define $T(f): Y \rightarrow R$ by (2.18). Since obviously $\hat{f} \in L(s X)$ (cf. [20, Theorem 4.1]), we have $\hat{t} \in L(E)$ and hence $T(f) \in L(Y)$. Evidently $T: L(X) \rightarrow L(Y)$ is a homomorphism.

2.19. Corollary. If $X$ is compact, every homomorphism $T: L(X) \rightarrow L(Y)$ with $T(1)=1$ is induced by a unique Lipschitz map $t: Y \rightarrow X$, and conversely.

Proof. Since $s X=X$, this follows from 2.17 and 2.16.

2.20. Corollary. A map $t: Y \rightarrow X$ is LS if and only if $t^{\prime}(L(X)) \subset L(Y)$.

Proof. 2.17 and 2.16.

2.21. Corollary. Two metrics $d_{1}$ and $d_{2}$ on a set $S$ are LS equivalent if and only if $L\left(S, d_{1}\right)=L\left(S, d_{2}\right)$.

2.22. Theorem. Let $t: Y \rightarrow X$ be LS and $t^{\prime}: L(X) \rightarrow L(Y)$ the induced homomorphism. Then $t^{\prime}$ is injective if and only if $t(Y)$ is dense in $X$, and $t^{\prime}$ is surjective if and only if $t$ is injective and $t^{-1}: t(Y) \rightarrow Y$ is LS.

Proof. The first part follows from the fact that $L(X)$ is a completely regular subset of $C(X)$. To prove the second part, let $A=t(Y)$, let $t_{1}: Y \rightarrow A$ be the LS map defined by $t$ and let $j: A \rightarrow X$ be the inclusion map. Then $t^{\prime}$ is the composite of $j^{\prime}: L(X) \rightarrow L(A)$ and $t_{1}^{\prime}: L(A) \rightarrow L(Y)$. We first assume that $t_{1}$ is an LS homeomorphism; then $t_{1}^{\prime}$ is an isomorphism. Hence, since $j^{\prime}$ is surjective, $t^{\prime}$ is so. We now assume that $t^{\prime}$ is surjective. Since $L(Y)$ separates points of $Y, t_{1}$ is bijective. Since $t_{1}^{\prime}$ is surjective and by the above also injective, $t_{1}^{\prime}$ is an isomorphism. Obviously $t_{1}^{-1}$ induces $\left(t_{1}^{\prime}\right)^{-1}$. Hence $t_{1}^{-1}$ is LS by 2.20 .

2.23. Theorem. Two complete metric spaces $X$ and $Y$ are LS homeomorphic if and only if the rings $L(X)$ and $L(Y)$ are isomorphic. Every LS homeomorphism induces an isomorphism and every isomorphism is induced by a unique LS homeomorphism.

Proof. Let $T: L(X) \rightarrow L(Y)$ be an isomorphism. Then $T$ induces a homeomorphism $T^{\circ}: s Y \rightarrow s X$. By $2.12 T^{\circ}$ defines a homeomorphism $t: Y \rightarrow X$. Then $T=t^{\prime}$ and $T^{-1}=\left(t^{-1}\right)^{\prime}$. Hence by $2.20 t$ is an LS homeomorphism. The converse result is obvious.

2.24. Remark. Lemma 2.16 sharpens [20, Corollary 3.7] and its proof is simpler; if $d$ is bounded, it is the same as [20, Proposition 3.4]. For compact $X$ and $Y$, 2.19 and 2.22 cover precisely [20, Theorem 5.1], and thus 2.23 in this case follows from [20, Theorem 5.1] (see also [20, Corollary 5.2]). For bounded $d_{1}$ and $d_{2}, 2.21$ 
coincides with [20, Corollary 3.5]. Fraser [5, 2.4] proved 2.21 more generally for pseudometrics.

2.25. Remark. The following properties of $\varphi \in s X$ are equivalent: (1) $\varphi \in X$; (2) $\varphi$ is continuous in the topology of pointwise convergence of $L(X)$; (3) $\varphi$ is continuous in the topology of compact convergence of $L(X)$. The implications $(1) \Rightarrow(2) \Rightarrow$ (3) are trivial and $(3) \Rightarrow(1)$ follows from 2.10 . It is now easy to see that if we consider $L(X)$ and $L(Y)$ in 2.17 with the topology of pointwise convergence (or compact convergence), then $T$ is continuous if and only if $t$ is an LS map $E \rightarrow X$ (in this case we may replace $\hat{f}$ in (2.18) by $f$ ). Thus a map $T: L(X) \rightarrow L(Y)$ is a homeomorphic isomorphism if and only if $T=t^{\prime}$ where $t: Y \rightarrow X$ is an LS homeomorphism. The similar results [3, 3.4, 3.5, and Corollary of 3.5] seem to be erroneous (let $\alpha=\beta=\mathrm{id}$; then the induced map $t: Y \rightarrow X$ is LS, but not necessarily Lipschitz).

2.26. Lemma. The following properties of a map $t: X \rightarrow Y$ are equivalent:

(1) $t$ is Lipschitz.

(2) If $Z$ is a metric space and $f: Y \rightarrow Z$ is Lipschitz, then ft is Lipschitz.

(3) $t^{\prime}(\operatorname{Lip}(Y)) \subset \operatorname{Lip}(X)$.

Proof. Trivially, $(1) \Rightarrow(2) \Rightarrow(3)$. To prove $(3) \Rightarrow(1)$, we define a linear map $T=t^{\prime}: \operatorname{Lip}(Y) \rightarrow \operatorname{Lip}(X)$ and introduce a norm $\|f\|=\max (|f(b)|, \operatorname{lip} f)$ on $\operatorname{Lip}(Y)$, where $b \in Y$ is fixed (we may assume $Y \neq \emptyset$ ), and a similar norm on $\operatorname{Lip}(X)$. Then Lip $(X)$ and Lip $(Y)$ are Banach spaces and in them convergence implies pointwise convergence. Since $T$ is continuous if we consider $\operatorname{Lip}(X)$ and $\operatorname{Lip}(Y)$ with the topology of pointwise convergence, $T$ is continuous by the closed graph theorem. Let $x, y \in X$ and define $f \in \operatorname{Lip}(Y)$ by $f(z)=d^{\prime}(z, t(y))-d^{\prime}(b, t(y))$. Then $d^{\prime}(t(x), t(y))=$ $|T(f)(x)-T(f)(y)|$. Since $\|f\| \leqq 1$, we obtain lip $t \leqq\|T\|$.

Lemma 2.26 implies the analogue $[12,5.4]$ of 2.21 .

2.27. Theorem. Consider $\operatorname{Lip}(X)$ and $\operatorname{Lip}(Y)$ with the topology of pointwise convergence (or compact convergence). Let $T: \operatorname{Lip}(X) \rightarrow \operatorname{Lip}(Y)$ be continuous and let $T$ define a homomorphism $L(X) \rightarrow L(Y)$. Then there are a unique set $E \subset Y$ with $d^{\prime}(E, Y \backslash E)>0$ and a unique Lipschitz map $t: E \rightarrow X$ such that

$$
T(f)(x)=\left\{\begin{array}{lll}
f(t(x)) & \text { if } & x \in E \\
0 & \text { if } & x \in Y \backslash E
\end{array} \quad(f \in \operatorname{Lip}(X)) .\right.
$$

Conversely, if $E=Y$ and $t$ is as above, then (2.28) defines a map $T$ having the above properties.

Proof. Let $T$ be given. Then by 2.25 there are a unique set $E \subset Y$ with $d^{\prime}(E, Y \backslash E)>0$ and a unique map $t: E \rightarrow X$ such that (2.28) holds for every $f \in L(X)$. 
Let $f \in \operatorname{Lip}(X)$. Since the sequence $(\max (\min (f, n),-n))$ in $L(X)$ converges to $f$ and since $T$ is continuous, (2.28) holds also for this $f$. Since then $f t=T(f) \mid E$ is Lipschitz, 2.26 implies that $t$ is Lipschitz. The proof of the converse part is omitted.

\section{Rings of LIP functions}

3.1. In this section we consider the rings $l(X)$ and $l^{*}(X)$. These have been studied by Su [24] and Scanlon [17]; by the following lemma Su's $L_{c}$-mappings, which are defined by the property (2) of the Lemma, are precisely the LIP maps.

3.2. Lemma. The following properties of a map $f: X \rightarrow Y$ are equivalent:

(1) $f$ is LIP.

(2) $f \mid K$ is Lipschitz for each compact subset $K$ of $X$.

(3) If $x \in X$, then $\sup _{n} d^{\prime}\left(f\left(y_{n}\right), f\left(z_{n}\right)\right) / d\left(y_{n}, z_{n}\right)<\infty$ for all sequences $\left(y_{n}\right)$ and $\left(z_{n}\right)$ in $X$ with $y_{n} \rightarrow x, z_{n} \rightarrow x$, and $y_{n} \neq z_{n}$ for each $n$.

(4) If $Z$ is a metric space and $g: Y \rightarrow Z$ is LIP, then $g f$ is LIP.

(5) $f^{\prime}(L(Y)) \subset l(X)$.

Proof. The implications $(1) \Rightarrow(2) \Rightarrow(3) \Rightarrow(1)$ are easy to verify; in $[17,2.1]$ these are proved for $Y=R$. In (3) the condition for $x$ is equivalent to the condition that $f$ is Lipschitz on some neighborhood of $x$. It is trivial that $(1) \Rightarrow(4) \Rightarrow(5)$. In [24, 5.4'] it is proved that (5) implies (2), but we repeat the proof here. Let $K \subset X$ be compact. Then $g(f \mid K)=(\bar{g} f) \mid K \in L(K)$ for each $g \in L(f(K))$, where $\bar{g} \in L(Y)$ denotes an extension of $g$. Thus $f \mid K$ is Lipschitz by 2.20 .

\subsection{Lemma. $l(X)^{\circ}=v X$.}

Proof. Here $v X$ is the Hewitt realcompactification of $X[6,8.8]$. Since $l(X)$ is a completely regular subring of $C(X)$, we may treat $X$ as a subspace of $C(X)^{\circ}$ and of $l(X)^{\circ}$ as in 2.3 ; then $C(X)^{\circ}=v X$ by $[6,11.8]$. We define the $m$-topology on $C(X)$ by taking as a neighborhood base at $g$ all sets $\{f|| f-g \mid \leqq u\}$, where $u \in C(X)$ is positive; then $C(X)$ is a topological ring $[6,2 \mathrm{~N} .1]$ and $l(X)$ is dense in $C(X)$ by $[14,5.18]$. Let $\varphi \in l(X)^{\circ}$. Since $l(X)$ is inverse-closed, $\varphi$ is continuous. Hence $\varphi$ has by uniform continuity a unique continuous extension $\alpha(\varphi)$ to $C(X)$, and $\alpha(\varphi) \in C(X)^{\circ}$. Since $C(X)^{\circ}$ is equicontinuous and $l(X)$ is dense in $C(X)$, it is easy to see that $\alpha: l(X)^{\circ} \rightarrow C(X)^{\circ}$ is a continuous bijection with $\alpha^{-1}(\varphi)=\varphi \mid l(X)$. Since $\alpha^{-1}$ is trivially continuous, $\alpha$ is a homeomorphism. Finally, $\alpha \mid X=\mathrm{id}$.

3.4. Remark. By 3.3 the $L_{c}$-realcompact metric spaces $X$ defined in $[24,4.3$ and 5.11] by $l(X)^{\circ}=X$ are precisely the realcompact metric spaces. Let $C^{k}(M)$ be the ring of all real-valued $C^{k}$ functions on a paracompact Hausdorff $C^{k}$ manifold $M$ (second countability not assumed), $k=1, \ldots, \infty$. It is proved in [11, Satz 3] as in 3.3 that $C^{k}(M)^{\circ}=M$ if and only if $M$ is realcompact. The proof of 3.3 shows that $C^{k}(M)^{\circ}=v M$ even if $M$ is not realcompact. 
3.5. Theorem. (Su) Two metric spaces $X$ and $Y$ are lipeomorphic if and only if $l(X)$ and $l(Y)$ are isomorphic. More precisely, every isomorphism is induced by $a$ unique lipeomorphism, and conversely.

Proof. Every lipeomorphism $t: Y \rightarrow X$ induces clearly an isomorphism $t^{\prime}: l(X) \rightarrow l(Y)$. Conversely, every isomorphism $T: l(X) \rightarrow l(Y)$ is of this form by $\left[24,6.4^{\prime}\right]$, since $T$ is linear by 2.2 and hence satisfies the additional condition of $\mathrm{Su}$ to leave all constant functions unchanged. Another proof is as follows. Since $T$ induces a homeomorphism $T^{\circ}: l(Y)^{\circ} \rightarrow l(X)^{\circ}$ and since $X=\left\{x \in v X \mid\{x\}\right.$ is a $G_{\delta}$-set $\}$ by [6, 9.7] and similarly for $Y$, by $3.3 t=T^{\circ} \mid Y$ is a homeomorphism $Y \rightarrow X$ with $T=t^{\prime}$. Then $t$ is a lipeomorphism by 3.2 .

3.6. Corollary. Two metric spaces $X$ and $Y$ are Lipschitz homeomorphic if and only if there is an isomorphism of $l(X)$ onto $l(Y)$ that carries $\operatorname{Lip}(X)$ onto $\operatorname{Lip}(Y)$.

Proof. 3.5 and 2.26 .

3.7. Lemma. $l^{*}(X)^{\circ}=\beta X$, where $\beta X$ is the Stone- Čech compactification of $X$.

Proof. As in 3.3, we may consider $X$ as a subspace of $C^{*}(X)^{\circ}$ and of $l^{*}(X)^{\circ}$, and then $C^{*}(X)^{\circ}=\beta X[6,11.9]$. The proof is now completed as in 3.3 , but instead of the $m$-topology the sup norm topology suffices.

3.8. Theorem. Two metric spaces $X$ and $Y$ are lipeomorphic if and only if $l^{*}(X)$ and $l^{*}(Y)$ are isomorphic. More precisely, every isomorphism is induced by a unique lipeomorphism, and conversely. $[6,9.7]$.

Proof. This follows from 3.7 and 3.2 because $X=\left\{x \in \beta X \mid\{x\}\right.$ is a $G_{\delta}$-set $\}$

3.9. Corollary. Two metric spaces $X$ and $Y$ are LS homeomorphic if and only if there is an isomorphism of $l^{*}(X)$ onto $l^{*}(Y)$ that carries $L(X)$ onto $L(Y)$.

Proof. 3.8 and 2.20.

3.10. Definition. Let the seminorm $p_{K}$ on $l(X)$ be defined for each compact set $K \subset X$ by $p_{K}(f)=\|f \mid K\|_{\infty}+\operatorname{lip}(f \mid K)$. The l-topology on $l(X)$ is defined by taking as a neighborhood base at $g$ all sets $\left\{f \mid p_{K}(f-g)<\varepsilon\right\}$, where $K \subset X$ is compact and $\varepsilon>0$.

3.11. Theorem. In the l-topology $l(X)$ is a complete Hausdorff multiplicativelyconvex topological algebra. The l-topology is given by a norm if and only if $X$ is compact, and then by $p_{X}$, i.e., (2.6). The space $l(X)$ is metrizable if and only if $X$ is locally compact and separable, and it is separable if and only if $X$ is discrete and card $X \leqq$ card $R$.

Proof. Since the $l$-topology is defined by the seminorms $p_{K}$ and $p_{K}(f g) \leqq$ $p_{K}(f) p_{K}(g)$, we conclude that $l(X)$ is a locally convex topological algebra and 
indeed a multiplicatively-convex one. Clearly $l(X)$ is Hausdorff. If $X$ is compact, obviously $p_{X}$ defines the $l$-topology.

To prove the completeness, let $\mathscr{F}$ be a Cauchy filter in $l(X)$. If $K \subset X$ is compact, the sets $\{f|K| f \in F\}, F \in \mathscr{F}$, in the Banach space $L(K)$ form a Cauchy filter base, which hence converges to a function $f_{K} \in L(K)$. We define $f: X \rightarrow R$ by $f(x)=$ $f_{\{x\}}(x)$. Then $f \mid K=f_{K}$ for every compact $K \subset X$, and therefore $f \in l(X)$ by 3.2. Obviously $\mathscr{F} \rightarrow f$.

The characterizations of normability and metrizability of $l(X)$ can be proved as the corresponding results for the topology of compact convergence on $C(S)$, $S$ a topological space, in [1, Theorem 13] and [1, Theorems 7 and 8], respectively.

Suppose that $X$ is not discrete. Then there is a nondiscrete compact set $K \subset X$. By [10, Theorem 1] the Banach space $L(K)$ contains a linear subspace isomorphic to $m(N)$, where $N$ is the set of positive integers, and is thus nonseparable. This implies that for each sequence $\left(f_{n}\right)$ in $l(X)$ there is $g \in l(X)$ with $\inf _{n} p_{K}\left(f_{n}-g\right)>0$, i.e., $l(X)$ is not separable.

We now suppose that $X$ is discrete. Then $l(X)=R^{X}$ and the $l$-topology is the topology of pointwise convergence. Hence by [4, VIII.7.2(3)] $l(X)$ is separable if and only if $\operatorname{card} X \leqq$ card $R$.

3.12. Finally we prove some results on ideals of $l(X)$ and $l^{*}(X)$ by using results on ideals of $C(S)$ and $C^{*}(S)$, where $S$ is a completely regular Hausdorff space, and the approximation theorem $[14,5.18]$. By ideal we mean proper ideal. If $A$ is a commutative ring with unity, let $M(A)$ be the set of all maximal ideals in $A$ considered with the topology in which $\bar{F}=\{M \in M(A) \mid M \supset \cap F\}$ for every $F \subset M(A)$ (cf. [6,7M]). If $f \in C(S)$, we denote $Z(f)=f^{-1}(0)$. If $f \in C^{*}(S)$, we let $f^{\beta}$ be the unique continuous extension of $f$ to $\beta S$.

We consider $C(S)$ with the $m$-topology and $C^{*}(S)$ with the sup norm. The topology on the ring $l(X)$ induced by $C(X)$ is intrinsic, because for every positive $u \in C(X)$ there is an invertible element $v$ of $l(X)$ with $v^{2} \leqq u$. Also the sup norm on $l^{*}(X)$ can be defined intrinsically. Since $l(X)$ and $l^{*}(X)$ are inverse-closed, the closure of every ideal is an ideal and hence every maximal ideal is closed. This is also true of $C(S)$ and $C^{*}(S)$; cf. [6,2M and 2N]. If $A$ is either $C(X), C^{*}(X)$, $l(X)$ or $l^{*}(X)$, we let $I(A)$ denote the set of all closed ideals in $A$; then $M(A) \subset I(A)$.

3.13. Theorem. The maps $\pi: I(C(X)) \rightarrow I(l(X)), I \mapsto I \cap l(X)$, and $\pi^{*}: I\left(C^{*}(X)\right) \rightarrow$ $I\left(l^{*}(X)\right), I \mapsto I \cap l^{*}(X)$, are bijections.

Proof. We first consider $\pi$. Since $l(X)$ is a dense subring of the topological ring $C(X)$, one can define a map $\mu: I(l(X)) \rightarrow I(C(X))$ by $\mu(I)=\bar{I}$. Clearly, $\pi \mu=$ id. In order to prove $\mu \pi=\mathrm{id}$, we show that if $I$ is an ideal in $C(X)$, then $I \subset \overline{I \cap l(X)}$. Let $f \in I$ and let $u \in C(X)$ be positive. By [6,70.3] there is $g \in C(X)$ such that $|g-f| \leqq u$ and $\operatorname{cl}_{\beta X} Z(g)$ is a neighborhood of $\operatorname{cl}_{\beta X} Z(f)$ in $\beta X$. Since by [14, 5.18] 
there is $h \in l(X)$ such that $|h-g| \leqq u$ and $Z(h) \supset Z(g)$, we may assume $g \in l(X)$. Since $g \in I$ by [6, 70.1 and 70.2], $f \in \overline{I \cap l(X)}$.

We now consider $\pi^{*}$. As above one can define a map $\mu^{*}: I\left(l^{*}(X)\right) \rightarrow I\left(C^{*}(X)\right)$ by $\mu^{*}(I)=\bar{I}$; then $\pi^{*} \mu^{*}=$ id. Let $I$ be an ideal in $C^{*}(X)$ and let $f \in I$ and $\varepsilon>0$. Since $k \mapsto k^{\beta}$ is an isomorphism of $C^{*}(X)$ onto $C(\beta X)$, by [6,4O.2] there is $g \in C^{*}(X)$ such that $\|g-f\|_{\infty}<\varepsilon$ and $Z\left(g^{\beta}\right)$ is a neighborhood of $Z\left(f^{\beta}\right)$ in $\beta X$. Since there is $h \in l^{*}(X)$ such that $\|h-g\|_{\infty}<\varepsilon$ and $Z(h) \supset Z(g)$, we may assume $g \in l^{*}(X)$. Since $g \in I$ by $[6,40.1], f \in \overline{I \cap l^{*}(X)}$. This implies $\mu^{*} \pi^{*}=\mathrm{id}$.

3.14. Corollary. The sets $M^{p}=\left\{f \in l(X) \mid p \in \mathrm{cl}_{\beta X} Z(f)\right\}, p \in \beta X$, are the maximal ideals in $l(X)$, and $p \mapsto M^{p}$ is a homeomorphism of $\beta X$ onto $M(l(X))$. An ideal in $l(X)$ is closed in the m-topology if and only if it is an intersection of maximal ideals.

Proof. Since $\pi$ in 3.13 preserves inclusion, it is easy to see that $\pi$ defines a homeomorphism $M(C(X)) \rightarrow M(l(X))$. The Corollary now follows from the similar characterizations of $M(C(X))$ and of the closed ideals in $C(X)$ given in [6, 7.3 and 7.11] and [6, 7Q.2], respectively.

3.15. Corollary. The sets $M^{* p}=\left\{f \in l^{*}(X) \mid f^{\beta}(p)=0\right\}, p \in \beta X$, are the maximal ideals in $l^{*}(X)$, and $p \mapsto M^{* p}$ is a homeomorphism of $\beta X$ onto $M\left(l^{*}(X)\right)$. An ideal in $l^{*}(X)$ is closed in the sup norm if and only if it is an intersection of maximal ideals.

Proof. Since $\pi^{*}$ in 3.13 defines a homeomorphism $M\left(C^{*}(X)\right) \rightarrow M\left(l^{*}(X)\right)$, the Corollary follows from the similar characterizations of $M\left(C^{*}(X)\right)$ and of the closed ideals in $C^{*}(X)$ given in [6, 7.2 and 7.10] and [6, 40.4], respectively.

3.16. Remark. Our methods in $3.13,3.14$, and 3.15 also apply to the ring $C^{k}(M)$ (see 3.4) and its subring $C^{k}(M) \cap m(M)$. The first part of 3.14 and the second part of 3.14 and 3.15 also follow from [2, 2.6 and 3.1] and [21, Theorem 1], respectively.

\section{Rings of LIP functions on locally compact metric spaces}

4.1. In this section $X$ and $Y$ are locally compact metric spaces. We study the rings $l_{0}(X)$ and $l_{00}(X)$. A continuous map is proper if the inverse image of every compact set is compact.

4.2. Lemma. If $U$ is a neighborhood of a compact subset $K$ of $X$, there is $f \in l_{00}(X)$ such that $f|K=1, f| X \backslash U=0$, and $0 \leqq f \leqq 1$.

4.3. Theorem. Let $A$ be either $l_{0}(X)$ or $l_{00}(X)$ with the sup norm. Then the closed ideals in $A$ are the sets $I_{E}=\{f \in A|f| E=0\}$, where $E$ is a nonvoid closed subset of $X$, determined uniquely by $I_{E}$. The maximal ideals are $I_{x}=I_{\{x\}}, x \in X$. An ideal is closed if and only if it is an intersection of maximal ideals. 
Proof. The last assertion follows from the others. The first assertion can be proved by aid of 4.2 precisely as the corresponding fact in [7, C.30] for the Banach algebra $C_{0}(S, C)$ of all complex-valued continuous functions on a locally compact Hausdorff space $S$ vanishing at infinity. The ideals $I_{x}$ are maximal because $A / I_{x}$ is isomorphic to $R$.

Let $I$ be an ideal in $l_{00}(X)$. Then $I \subset I_{x}$ for some $x \in X$, because otherwise $I \supset l_{00}(X)$, as the proof of [7, C.30] shows. Thus $I=I_{x}$ if $I$ is maximal.

Let $I$ be a maximal ideal in $A=l_{0}(X)$. To prove that $I=I_{x}$ for some $x \in X$, we assume the contrary case and show modifying the proof for $C_{0}(S, C)$ in $[8,20.52(\mathrm{~d})]$ that this leads to a contradiction. Since $I \leftarrow I_{x}$ for each $x \in X, I \supset l_{00}(X)$ as above. If the ring $B=A / I$ is a field, let $f$ be a function in $A$ such that $f+I$ is the unity of $B$. There is $g \in l_{00}(X)$ with $\|f-g\|_{\infty}<1$ as follows from 4.2 ; then $h=f /(1-f+g) \in A$ and $f=h-h f+h g$. Since $h-h f \in I$ and $h g \in l_{00}(X) \subset I$, this implies $f \in I$, a contradiction. Thus all products in $B$ are zero; cf. [16, 3.1.1]. Let $f \in A$. By [4, XI.7.3] there are disjoint open subsets $U$ and $V$ of $X$ such that $X=U \cup V$, $U$ is separable, and $f \mid V=0$. There is a positive continuous function $\varphi$ on $U$ vanishing at infinity. By $[14,5.4]$ one may assume that $\varphi$ is LIP. We define $\psi \in A$ by $\psi \mid U=\varphi$ and $\psi \mid V=0$. Then $g=(f+|f|+\psi)^{1 / 2}$ and $h=(|f|+\psi)^{1 / 2}$ belong to $A$, and $f=g^{2}-h^{2}$. This implies that $I=A$, a contradiction.

\subsection{Corollary. Let $A$ be either $l_{0}(X)$ or $l_{00}(X)$. Then $A^{\circ}=X$.}

Proof. It follows from 4.2 that $x \mapsto \varphi_{x}$ is a topological embedding of $X$ into $A^{\complement}$. If $\varphi \in A^{\circ}$, then, since $\varphi$ is linear, the $\operatorname{ring} A / \operatorname{ker} \varphi$ is isomorphic to $R$, and hence $\operatorname{ker} \varphi$ is a maximal ideal. Thus by 4.3 there is $x \in X$ with $\operatorname{ker} \varphi=\operatorname{ker} \varphi_{x}$, whence $\varphi=\varphi_{x}$.

Corollary 4.4 also follows from [16, 3.2.8].

4.5. Lemma. Let $t: Y \rightarrow X$ and $t^{\prime}\left(l_{00}(X)\right) \subset l_{0}(Y)$. Then $t$ is a proper LIP map.

Proof. Since $l_{00}(X)$ is a completely regular subset of $C(X), t$ is continuous. If $K \subset X$ is compact, there is $f \in l_{00}(X)$ with $f \mid K=1$, and then $t^{-1}(K)$ is compact as a closed subset of the compact set $(f t)^{-1}(1)$. Thus $t$ is proper. Let $x \in Y$ and choose a neighborhood $U$ of $x$ such that $K=\overline{t(U)}$ is compact. If $f \in L(K)$, there are $g \in L(X)$ and $h \in l_{00}(X)$ with $g \mid K=f$ and $h \mid K=1$; then $\vec{f}=g h \in l_{00}(X)$ and $\bar{f} \mid K=f$, whence $f(t \mid U)=(\bar{f} t) \mid U$ is LIP. Thus $t \mid U$, and hence also $t$, is LIP by 3.2 .

4.6. Theorem. Let $T: l_{00}(X) \rightarrow l_{00}(Y)$ be a homomorphism. Then there are a unique open subset $E$ of $Y$ and a unique proper LIP map $t: E \rightarrow X$ such that

$$
T(f)(x)=\left\{\begin{array}{lll}
f(t(x)) & \text { if } & x \in E \\
0 & \text { if } & x \in Y \backslash E
\end{array} \quad\left(f \in l_{00}(X)\right) .\right.
$$

Conversely, if $E$ and $t$ are as above, then (4.7) defines a homomorphism $T: l_{00}(X) \rightarrow$ $l_{00}(Y)$. 
Proof. Let $T$ be given. Then $E=\left\{x \in Y \mid T(f)(x) \neq 0\right.$ for some $\left.f \in I_{00}(X)\right\}$ is open, and by 4.4 there is a map $t: E \rightarrow X$ such that (4.7) holds. If $f \in l_{00}(X)$ and $\varepsilon>0$, then $\{x \in E|| f(t(x)) \mid \geqq \varepsilon\}=\{x \in Y|| T(f)(x) \mid \geqq \varepsilon\}$ is compact, whence $f t \in l_{0}(E)$ (in fact, $f t \in l_{00}(E)$ ). Thus $t$ is a proper LIP map by 4.5. The rest of the proof is omitted.

4.8. Corollary. Two locally compact metric spaces $X$ and $Y$ are lipeomorphic if and only if $l_{00}(X)$ and $l_{00}(Y)$ are isomorphic.

4.9. Theorem. Theorem 4.6 holds if " $l_{00}$ " is replaced by " $l_{0}$ " and "open" by "open and closed".

Proof. It is clear that if $E \subset Y$ is open and closed and $t: E \rightarrow X$ is LIP and proper, then (4.7) where $f \in l_{0}(X)$ defines a homomorphism $T: l_{0}(X) \rightarrow l_{0}(Y)$. Conversely, let $T: l_{0}(X) \rightarrow l_{0}(Y)$ be a homomorphism. Then the proof of 4.6 shows that there are a unique open subset $E$ of $Y$ and a unique proper LIP map $t: E \rightarrow X$ such that (4.7) holds for all $f \in l_{0}(X)$. We complete the proof by showing that $E$ is closed. Assume the contrary case. Then there are $x \in Y \backslash E$ and a sequence $\left(x_{n}\right)$ in $E$ with $x_{n} \rightarrow x$. Since $t$ is proper, the sequence $y_{n}=t\left(x_{n}\right)$ in $X$ tends to infinity. One may assume that $y_{m} \neq y_{n}$ if $m \neq n$. Then it is easy to construct $f \in l_{0}(X)$ such that $f\left(y_{n}\right)=d^{\prime}\left(x_{n}, x\right)^{1 / 2}$ for every $n$. But $\left|T(f)\left(x_{n}\right)-T(f)(x)\right| / d^{\prime}\left(x_{n}, x\right)=d^{\prime}\left(x_{n}, x\right)^{-1 / 2}$, which contradicts the fact that $T(f)$ is LIP.

4.10. Corollary. Two locally compact metric spaces $X$ and $Y$ are lipeomorphic if and only if $l_{0}(X)$ and $l_{0}(Y)$ are isomorphic.

4.11. Remarks. 1) We did not use the local compactness of $Y$ in $4.5,4.6$ or 4.9. However, if one gives up this assumption, then, nevertheless, $Y$ in 4.5 and $E$ in 4.6 and 4.9 are always locally compact.

2) The results of this section still hold if the category of locally compact metric spaces and LIP maps is replaced either by the category of locally compact Hausdorff spaces and continuous maps or by the category of $C^{k}$ manifolds (in the sense of 3.4) and $C^{k}$ maps. The only exception is that $E$ in 4.9 in the case of $C_{0}(S)\left(=C_{0}(S, C) \cap R^{S}\right)$ need not be closed. The proofs are similar. The characterizations of homomorphisms seem to be new results; I know only the result $[18$, Zusatz 1] in this direction. The characterizations of isomorphisms are known.

\section{References}

[1] Arens, R.: A topology for spaces of transformations. - Ann. of Math. 47, 1946, 480-495.

[2] Biles, C. M.: Gelfand and Wallman-type compactifications. - Pacific J. Math. 35, 1970, 267278.

[3] Bishop, E. R.: Generalized Lipschitz algebras. - Canad. Math. Bull. 12, 1969, 1-19.

[4] Dugundu, J.: Topology. - Allyn and Bacon, Inc., Boston, 9th printing, 1974. 
[5] Fraser, R. B.: Banach spaces of functions satisfying a modulus of continuity condition. Studia Math. 32, 1969, 277-283.

[6] Gillman, L., and M. Jerison: Rings of continuous functions. - D. Van Nostrand Co., Inc., Princeton, N. J.-Toronto-New York-London, 1960.

[7] Hewitt, E., and K. A. Ross: Abstract harmonic analysis. Vol. I. - Springer-Verlag, BerlinGöttingen-Heidelberg, 1963.

[8] Hewitt, E., and K. Stromberg: Real and abstract analysis. - Springer-Verlag, Berlin-Heidelberg-New York, 1965.

[9] Johnson, J. A.: Banach spaces of Lipschitz functions and vector-valued Lipschitz functions. Trans. Amer. Math. Soc. 148, 1970, 147-169.

[10] Johnson, J. A.: Lipschitz spaces. - Pacific J. Math. 51, 1974, 177-186.

[11] Karrer, G., and R. MÜHLEThaler: Reelle Funktionenringe. - Topics in analysis. Colloquium on mathematical analysis, Jyväskylä 1970. Ed. O. Lehto, I. S. Louhivaara and R. Nevanlinna. Springer-Verlag, Berlin-Heidelberg-New York, 1974, 232-236.

[12] KatĚtov, M.: On quasi-metric properties. - Studia Math. (Ser. Specjalna) Zeszyt 1, 1963, 57-68 (Russian).

[13] Loomis, L. H.: An introduction to abstract harmonic analysis. - D. Van Nostrand Co., Inc., Princeton, N. J.--Toronto-New York-London, 1953.

[14] Luukkainen, J., and J. VÄIsÄLÄ: Elements of Lipschitz topology. - Ann. Acad. Sci. Fenn. Ser. A I 3, 1977, 85-122.

[15] McShane, E. J.: Extension of range of functions. - Bull. Amer. Math. Soc. 40, 1934, 837842.

[16] Rickart, C. E.: General theory of Banach algebras. - D. Van Nostrand Co., Inc., Princeton, N. J.-Toronto-New York-London, 1960.

[17] Scanlon, C. H.: Rings of functions with certain Lipschitz properties. - Pacific J. Math. 32, 1970, 197-201.

[18] Schirmeier, U.: Ein Banach—Stone-Satz für adaptierte Vektorverbände und Algebren. - Math. Z. $156,1977,279-290$.

[19] Semadeni, Z.: Banach spaces of continuous functions. Vol. I. - PWN-Polish Scientific Publishers, Warsaw, 1971.

[20] Sherbert, D. R.: Banach algebras of Lipschitz functions. - Pacific J. Math. 13, 1963, 13871399.

[21] Shirota, T.: On ideals in rings of continuous functions. - Proc. Japan Acad. 30, 1954, 85-89.

[22] Smirnov, Ju. M.: On proximity spaces. - Amer. Math. Soc. Transl. (2) 38, 1964, 5-35.

[23] Smirnov, Ju. M.: On the completeness of proximity spaces. I. - Ibid. 37-73.

[24] SU, LI PI: Algebraic properties of certain rings of continuous functions. - Pacific J. Math. 27, 1968, 175-191.

University of Helsinki

Department of Mathematics

SF-00100 Helsinki 10

Finland

Received 22 February 1978

Revision received 25 May 1973 